\title{
A smartphone microscope method for simultaneous detection of (oo)cyst of Cryptosporodium and Giardia
}

Retina Shrestha ${ }^{1}$, Rojina Duwal ${ }^{1}$, Sajeev Wagle ${ }^{1}$, Samiksha Pokhrel ${ }^{1,2}$, Basant Giri ${ }^{1, *}$, Bhanu

Bhakta Neupane ${ }^{1,3, *}$

${ }^{1}$ Centre for analytical sciences, Kathmandu Institute of Applied Sciences, Kathmandu, Nepal

${ }^{2}$ Department of Environmental Science, Tri-Chandra Multiple Campus, Tribhuvan University,

Kathmandu, Nepal

${ }^{3}$ Central Department of Chemistry, Tribhuvan University, Kathmandu, Nepal

Corresponding email:

BBN: bbneupane@cdctu.edu.np

BG: bgiri@kias.org.np 


\begin{abstract}
Gastrointestinal disorders caused by ingestion of (oo)cysts of Cryptosporodium and Giardia is one of the major health problems in developing countries. We developed a smartphone based microscopic assay method to screen (oo)cysts of Cryptosporodium and Giardia contamination in vegetable and water samples. We used sapphire ball lens as the major imaging element to modify a smartphone as a microscope. Imaging parameters such as field of view and magnification, and image contrast under different staining and illumination conditions were measured. The smartphone microscope method consisting of ball lens of $1 \mathrm{~mm}$ diameter, white LED as illumination source and Lugols's iodine staining provided magnification and contrast capable of distinguishing (oo)cysts of Crypstopsporodium and Giardia in the same sample. The analytical performance of the method was tested by spike recovery experiments. The spiking recovery experiments performed on cabbage, carrot, cucumber, radish, tomatoes, and water resulted $26.8 \pm 10.3,40.1 \pm 8.5,44.4 \pm 7.3,47.6 \pm 11.3,49.2 \pm 10.9$, and $30.2 \pm 7.9 \%$ recovery for Cryptosporodium, respectively and 10.2 $\pm 4.0,14.1 \pm 7.3,24.2 \pm 12.1,23.2 \pm 13.7,17.1 \pm 13.9$, and $37.6 \pm 2.4 \%$ recovery for Giardia, respectively. These recovery results were found to be similar when compared with the commercial brightfield and fluorescence microscopes. We tested the smartphone microscope system for detecting (oo)cysts on 7 types of vegetable $(n=196)$ and river water $(\mathrm{n}=18)$ samples. Forty two percent vegetable and thirty-nine percent water samples were found to be contaminated with Cryptosporodium oocyst. Similarly, thirty one percent vegetable and thirty three percent water samples were contaminated with Giardia cyst. This study showed that the developed method can be a cheaper alternative for simultaneous detection of (oo)cysts in vegetable and water samples.
\end{abstract}

Keywords: Interoparasites, low-cost microscope, waterborne disease, diarrhoea 


\section{Introduction}

Food and waterborne illnesses arising from the consumption of contaminated food and water are serious health hazards globally [1]. The World Health Organization (WHO) has reported 1.5 billion episodes of diarrhoeal cases leading to 3.5 million deaths of under 5-year-old children in developing countries annually. More than $70 \%$ of these diarrhoeal episodes are attributable to biologically contaminated food [2]. In order to prevent and identify the disease, detection of food borne parasite is important at all levels of production chain followed by screening and certification [3].

Cryptosporidium and Giardia are the major food and water-borne parasites [4]. Ninety percent of reported outbreaks of these pathogenic protozoans occur through water, while $10 \%$ are related to food. In the infective stage, Cryptosporidium oocysts have spherical shape with a diameter of 4-6 $\mu \mathrm{m}$ and Giardia cysts have elliptical shape of 8-12 um long and 7-10 $\mu \mathrm{m}$ wide [5]. Both of the cysts, collectively termed as (oo)cysts, have a simple and direct life cycle, which is extremely suitable for transmission by fresh produce. Additionally, the cysts are small in size with a robust transmission stage. Some genotypes of the parasites even have zoonotic potential giving the opportunity for contamination to occur from both animal and human sources. Cryptosporidia are particularly threatening as they are resistant to chlorine disinfection, can persist in the environment for a long period, can infect other animal hosts, and are difficult to diagnose and treat. The infectious dose for Giardia cysts and Cryptosporidium oocysts are 10 100 and 10-1000 respectively, which makes these pathogens more precarious [6]. Developing countries are the most vulnerable countries to these protozoans where infection is more likely underdiagnosed and underreported, and has limited resources and infrastructures for investigation [7]. In low income countries, the overall prevalence rate of Giardia infection is 20 - 30\% and the occurrence of Cryptosporidium are $4-31 \%$ in children younger than 10 years $[8]$.

Several methods have been described to detect Giardia cyst and Cryptosporidium oocyst in food, water, and faecal samples with high sensitivity and specificity. Commonly used approaches are polymerase chain reaction, flow cytometry, optical microscopic examination etc. However, these techniques need a good laboratory facility, well trained user and are expensive, therefore are not appropriate for low-resource settings including remote and field sites. There is a need for a simple, easy to use, rapid but reliable and low-cost test method for the detection of parasites [9-12]. 
In recent years, smartphone based systems are being explored and used as an alternative platform for the detection of microscopic to sub-microscopic specimens in a wide variety of matrices, such as parasite eggs in faecal sample[13], allergen in food [14], blood cells in blood [15], single nanoparticles and viruses [16], filarial and malarial parasites in blood [17, 18], sickle cell anaemia in a blood smear [19], soil-transmitted helminth and fluke in urine and stool samples [20] etc.

In this work, we describe a smartphone microscopic system that can image and quantify (oo)cysts of both Cryptosporidium and Giardia in a given sample. We optimized optical parameters of the microscope including field of view, magnification, and image contrast under different staining and illumination conditions. The validity of the developed microscope was tested by spiking the vegetable sample with known number of standard (oo) cyst samples. For comparison, the spiked vegetable samples were also imaged with a commercial bright field and a fluorescence microscope and the percentage recovery data were compared. The optimized smartphone microscope was also used to measure (oo)cyst contamination in vegetable, fruits and water samples.

\section{Materials and methodology}

\section{Design of smartphone microscope}

We used a sapphire ball lens (Edmund Optics, New Jersey, USA) and a mounting plate made up of aluminium to transform a smartphone (Samsung Galaxy J7 prime) into a smartphone microscope. A small hole was punctured at the centre of the mounting plate and the ball lens was firmly glued in this hole (figure 1A). The ball lens was then centred over the smartphone camera lens. The mounting plate was fixed onto the smartphone using a transparent tape. The smartphone had a rare camera of $13 \mathrm{MP}$ and the screen size of the phone was $151.7 \mathrm{~mm} \times 75$ mm with 1080 x 1920 pixels resolution. We custom built a microscope stage to hold the sample slide using a wooden viewing box of dimension $15 \times 15 \mathrm{~cm}$. A $3 \mathrm{~cm}$ diameter hole was drilled on top centre position of the box to have the illumination light pass through the sample specimen placed on the microscopic slide just above the hole. The slide was fixed on both side of the hole using a double-sided tape. A schematic of the optical set up is shown in figure 1. 

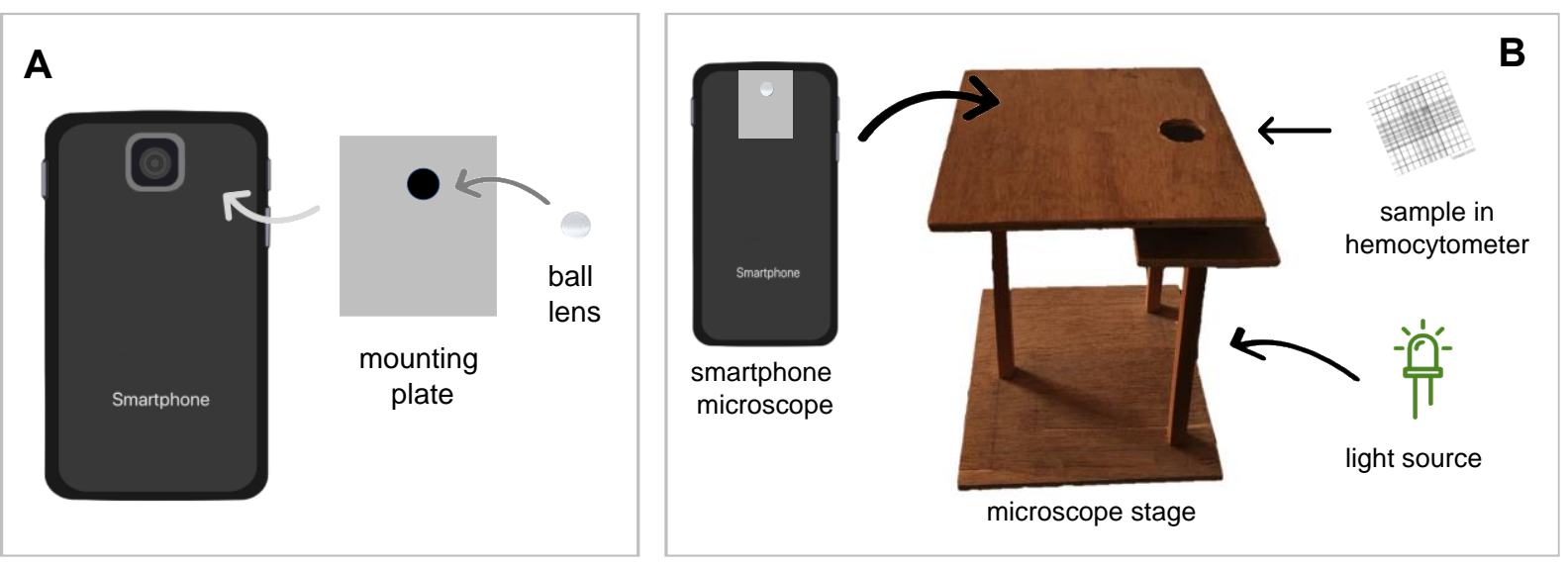

Figure 1: Assembly of smartphone microscope (A) and a measurement set up (B).

\section{Measurement of magnification and contrast}

We tested three ball lenses having different diameter of $0.5 \mathrm{~mm}, 1 \mathrm{~mm}$, and $2 \mathrm{~mm}$. Image of a standard calibration grid (Generic, USA; 1 division $=10 \mu \mathrm{m}$ ) was captured with the ball lens attached to the smartphone. The distance covered by all the grid lines was measured in pixel using ImageJ software. The pixel distance was converted to micrometre to get field of view (FOV) of the microscopic system.

The magnification $(M A G)$ was calculated as:

$$
M A G=\frac{b}{a}
$$

Where, $a$ and $b$ are size of object and image, respectively.

To measure the contrast, we imaged standard (oo)cysts sample (Waterborne TM, Inc., New Orleans, USA) under different illumination and staining conditions to determine the image contrast. Based on field of view and magnification, we chose $1 \mathrm{~mm}$ ball lens in our further experiments. We tested two different light sources for sample illumination: a smartphone flashlight and a white light emitting diode (LED, 12 watt). We also tested two different types of stains: Lugol's iodine (HiMedia, Mumbai, India) and methylene blue dye (Fisher Scientific, New Hampshire, United States). The staining experiments involved a mixture of Cryptosporodium parvum and Giardia intestinalis (oo) cysts suspension $\left(\sim 2 \times 10^{5}\right.$ (oo) cyst per $\mathrm{mL}$ ) and stain dye in 1:1 ratio. An aliquot of $10 \mu \mathrm{L}$ of the mixture was loaded on the Haemocytometer (Max Levy, Philadelphia, USA) and was then illuminated by the light source and viewed through smartphone microscope. For each light source, images of (oo)cysts were captured at different time intervals after mixing the dye with the standard (oo)cysts. The images 
were analysed in ImageJ software to calculate the Weber contrast in percentage (WC) as follows [21]:

$$
W C=\left(\frac{I-I_{b}}{I_{b}}\right) 100
$$

where, $I$ is the intensity on the specimen of interest i.e. (oo) cyst and $I_{b}$ is the average intensity of immediately adjacent background.

\section{Spike recovery experiments}

Five different types of vegetables such as tomato, cabbage, carrot, radish, and cucumber were selected for spike recovery experiments. These vegetables were selected based on previous reports of faecal contamination [22-25]. These vegetables are consumed in raw forms in many countries. All the samples were bought from the local vegetable shops. A portion of the sample (15-20 g) was soaked in distilled water for about 20 minutes to remove all the surface contaminants including (oo)cysts. After washing the samples, $10 \mu \mathrm{L}$ of a mixture of $C$. parvum oocysts and G. intestinalis cyst suspension (Waterborne Inc, PC101 G/C positive control) was added to randomly selected points on sample surface samples with a micropipette. The (oo)cysts seeded sample was left to dry at room temperature for 2 hours to overnight.

We also performed similar spike recovery experiments with water samples. Five sets of $50 \mathrm{~mL}$ distilled water $\left(\mathrm{pH}=6.8\right.$, conductivity $\left.=0.01 \mathrm{Sm}^{-1}\right)$ were spiked with $10 \mu \mathrm{L}$ of the standard (oo)cyst suspension and were incubated overnight.

We tested three different washing solutions such as distilled water, normal saline, and glycine buffer (1M, pH 5.5) to extract (oo)cysts from each spiked vegetable samples The sample was put in extracting solution in a ziplock bag (Great Value, Fresh Seal Double Zipper). Ziplock bag was used as a low-cost alternative to stomacher bag. The eluate was then carefully transferred into two $50 \mathrm{~mL}$ falcon tubes. The elute suspension was concentrated by using the triple centrifugation method proposed by Medeiros and Daniel [26] with some modifications. At first eighty millilitres of eluate was centrifuged in two $50 \mathrm{~mL}$ tubes at $1500 \mathrm{x}$ g for 10 minutes. The supernatant was decanted into a clean beaker leaving a final volume of $5 \mathrm{~mL}$, which was placed in a vortex mixture for 20 seconds to homogenize the pellet. The $5 \mathrm{~mL}$ residual volume from each centrifugation tubes were combined together into a single tube. Another centrifugation was carried out at $1500 \mathrm{x} \mathrm{g}$ for 10 minutes. The supernatant was discarded leaving $0.5 \mathrm{~mL}$ pellet in the centrifuge tube. The residual solution was again vortexed for 20 seconds and it was carefully transferred to $1.5 \mathrm{~mL}$ microcentrifuge tube with $10 \mu \mathrm{L}$ 
micropipette. The centrifuge tube was rinsed with $0.5 \mathrm{~mL}$ distilled water and added to the same $1.5 \mathrm{~mL}$ microcentrifuge tube to make the final volume of $1 \mathrm{~mL}$. Now, the third centrifugation was performed at $1500 \mathrm{x} g$ for 10 minutes. The supernatant was removed, leaving just $0.5 \mathrm{~mL}$ in the microcentrifugation tube one more time.

The water samples containing (oo)cysts were subjected to flocculation and sedimentation as described by Karanis and Kimura [27] with some modifications. $50 \mathrm{~mL}$ of ferric sulphate $(0.25 \mathrm{M})$ solution was added to $50 \mathrm{~mL}$ of water samples and the $\mathrm{pH}$ was adjusted to $6 \pm 0.05$. The sample was left 24 hours at room temperature to precipitate floc. Then the supernatant was carefully aspirated with a syringe filter without disturbing the sediment. The sediment was further centrifuged at $2,000 \times g$ for 10 minutes and the supernatant was discarded. The pellet was dissolved in $1 \mathrm{~mL}$ of citric acid lysis buffer $(8.4 \mathrm{~g}$ citric acid monohydrate, $17.64 \mathrm{~g}$ trisodium-citrate-dihydrate, distilled $\mathrm{H}_{2} \mathrm{O}$ up to $100 \mathrm{~mL}$; $\mathrm{pH}$ 4.7) by incubating at room temperature for 1 hour with vortexing every 15 minutes. The sample was washed twice with distilled water by centrifugation at $2000 \times g$ for 10 minutes. The pellets collected were resuspended in $5 \mathrm{~mL}$ distilled water for the purification of (oo)cyts. The purification step is only required with contaminated water, whereas non-contaminated water can be pelleted followed by dissolving in the buffer and subjected to the microscopy.

The purification steps of water samples involved a discontinuous sucrose gradient. The gradient was prepared with the Sheather's solution ( $320 \mathrm{~mL} \mathrm{H}_{2} \mathrm{O}$ and $500 \mathrm{~g}$ sucrose) diluted with 0.025 M phosphate-buffered saline (PBS) and supplemented with 1\% Tween 80 to make 1:2 solution of 1.103 specific gravity and 1:4 solution of 1.064 specific gravity. $10 \mathrm{~mL}$ of 1:4 solution was layered over $10 \mathrm{~mL}$ of $1: 2$ solution on a $50 \mathrm{~mL}$ centrifuge tube. Then, $5 \mathrm{~mL}$ of the sample were layered over 1:4 solution and was centrifuged at $1500 \times g$ for 30 minutes. The three layers were recovered carefully and pooled separately along with the pellet and examined for the oocysts. The pooled layers were diluted with water, centrifuged and pellets were collected for the microscopic analysis.

\section{Microscopic measurements}

Ten microliters of each concentrated sample were stained with $10 \mu \mathrm{L}$ of diluted Lugol's iodine (1:2 in water) and subsequently loaded into hemocytometer. The sample was incubated for around 6 minutes. The (oo)cysts were screened and enumerated in four quadrants of the hemocytometer under smartphone microscope. The cysts on the same hemocytometer were simultaneously counted by brightfield microscope. Triplicate measurement was made for each concentrated suspension. 
The spiked samples were also examined with a fluorescent microscope (Labomed Inc, United States, LB 702). For fluorescence measurement, $5 \mu \mathrm{L}$ of (oo)cyst suspension was placed on the clean glass slide to which a drop of fluorescein-labelled mouse monoclonal antibodies (Aqua$\mathrm{Glo}^{\mathrm{TM}} \mathrm{G} / \mathrm{C}$ ) was applied. The slide was incubated at $37^{\circ} \mathrm{C}$ for 10 minutes in an incubator (Faithful, China) and imaged with $480 \mathrm{~nm}$ excitation and $520 \mathrm{~nm}$ emission wavelengths.

The numbers of (oo)cyst were counted before each seeded experiment using all three microscopic methods. The mean percentage recovery efficiency (RE) was calculated as:

$$
R E=\left(\frac{n}{N}\right) 100
$$

Where, $N$ is the number of (oo) cysts added to a sample and $n$ is the number of (oo) cysts recovered from the sample.

We followed the above procedure excluding the washing of raw vegetables and spiking with (oo)cyst to determine the (oo) cyst contamination in vegetable and water samples.

\section{Results and discussion}

\section{Optimization of smartphone microscope}

The performance of an imaging system is determined by its optical parameters, such as field of view, magnification, resolution, and contrast. The FOV is the size of the viewing area that can be sees when we look through a microscope. Magnification measures the zooming of an object, and resolution and contrast measure the details and clarity in an image [28]. The FOV of ball lens based imaging system depends on the size of ball lens, the refractive index of ball lens material and wavelength of illumination source; the size factor being major contributor [29]. On the other hand, the magnification of smartphone microscope depends on size of ball lens and also on the nature of smartphone that contains a built-in lens and CMOS camera at fixed distance. The spherical ball lens has a curved surface that results in curvature effect. It means the central region is more sharp/clear than the periphery in the image plane. The clear field of view microscope having ball lens of $0.5,1$, and $2 \mathrm{~mm}$ ball lens is provided in table 1 .

Table 1: Field of view (FOV) of the smartphone microscope

\begin{tabular}{cc}
\hline $\begin{array}{c}\text { Diameter of ball lens } \\
(\mathrm{mm})\end{array}$ & $\begin{array}{c}\text { Clear field of view } \\
(\mu \mathrm{m})\end{array}$ \\
\hline 0.5 & $114 \pm 5.6$ \\
1 & $203 \pm 5.7$ \\
2 & $638.7 \pm 15.9$ \\
\hline
\end{tabular}


The measured field of view (FOV) of smartphone microscope showed that the FOV increases with an increase in the diameter of ball lens (Table 1) which is in consistent with reported values [29].

The magnification of smartphone microscope with $1 \mathrm{~mm}$ ball lens was estimated to be $200 \mathrm{x}$. The $1 \mathrm{~mm}$ lens set up was able to magnify Cryptosporodium oocyst to $0.8-1.2 \mathrm{~mm}$ and Giardia cyst to 1.4-2.4 mm, respectively. The magnification was enough to distinguish the two specimens. Therefore, we selected a smartphone microscopic system having $1 \mathrm{~mm}$ diameter ball lens for further experiments in this study.

Light source used for illuminating sample affects the image quality of (oo) cyst. We tested following two types of commonly available light sources: white LED and smartphone light. The images collected using these light sources are shown in figure 2.
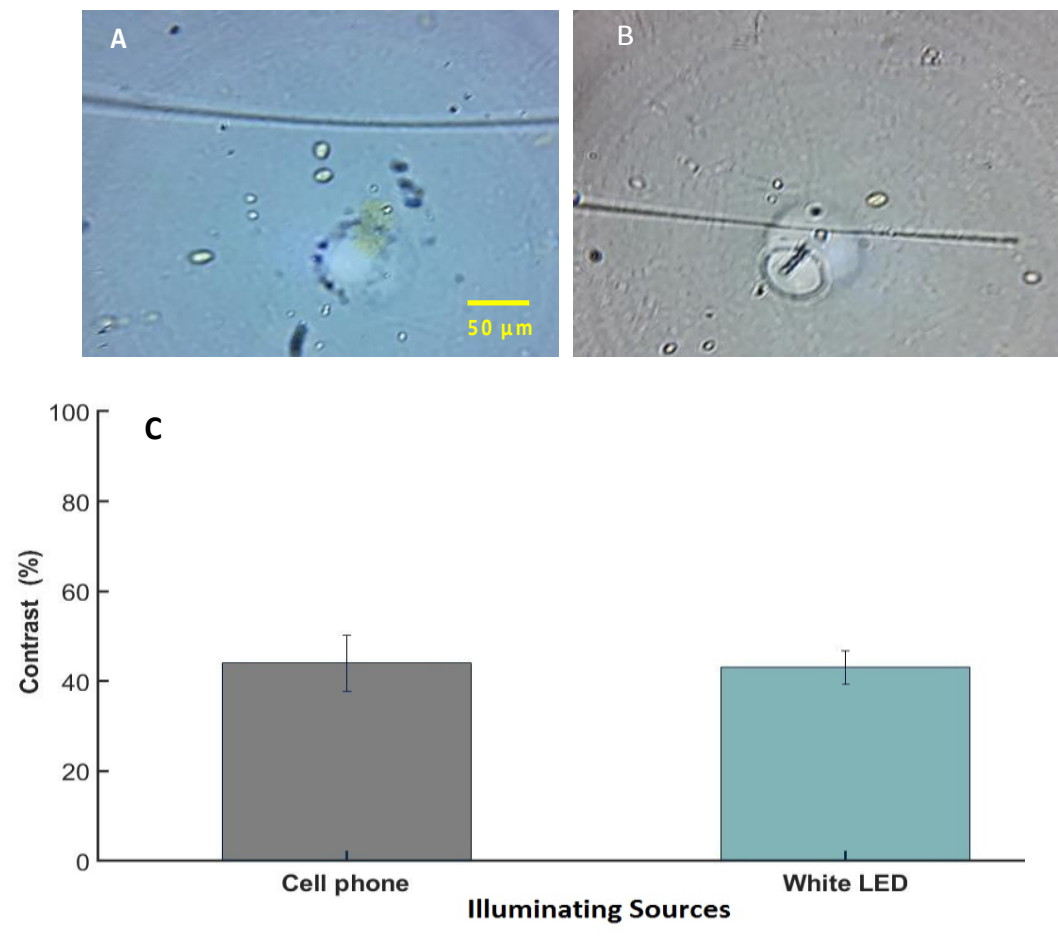

Figure 2: Photographs of (oo)cysts of cryptosporidium and giardia taken using smartphone microscope with $1 \mathrm{~mm}$ ball lens with (A) white LED light illumination and (B) smartphone flashlight illumination. Scale bar in both images is $50 \mu \mathrm{m}$. (C) The measured contrast for (oo)cysts. The error bar in $\mathrm{C}$ represent the standard deviation of triplicate measurements.

Images shown in figure 2 clearly depicts that the oocyst and cyst can be easily distinguished from each other based on their shape and size. Oocysts are circular shape and cysts are oval in 
shape. The Weber contrast for the (oo)cysts are shown in figure 2C. Both of the light sources provided similar contrast percentages. Since the white LED is easily available, cheaper, and easy to use, we chose it for further experiments.

A number of staining procedures have been developed to aid in the clear morphological identification and differentiation of (oo)cysts by light microscopy [9]. Some of the most used techniques are the iodine and methylene blue mounts. These methods are simple, faster and inexpensive and provide clear distinction of (oo)cyst by morphological features [10]. The temporal variation of stain color intensity on the cysts are shown in figure 3 . This shows the color intake by the cysts and stability of the stains with waiting time.

It is evident that Luglo's iodine (LI) provided brighter image than Methylene blue (MB). Also, LI staining, intensity increased after 2 minutes of incubation and remained constant for up to 12 minutes. This indicated that LI staining is more stable over time. Based on stability of stain and intensity, we selected LI staining and 6-8 minutes of staining time in the subsequent experiments. The Lugol's iodine staining also provided higher contrast compared to methylene blue dye staining. Lugol's iodine initially has contrast of $48 \pm 16.4 \%$ which decreased to $35.5 \pm 11.6 \%$ in next 2 minutes and remained constant throughout the time. In case of methylene blue contrast remained constant around $27.7 \%$ during the experiment. 
A
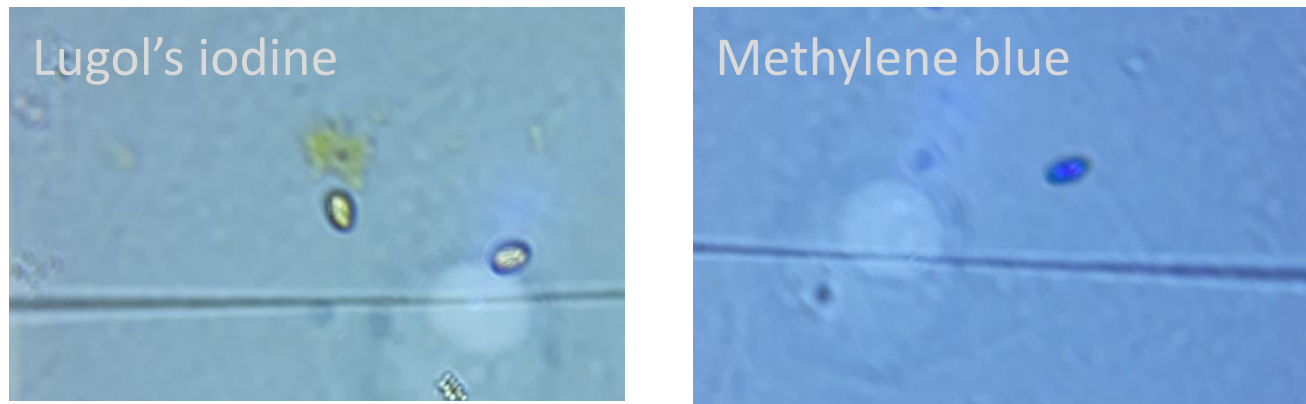

B

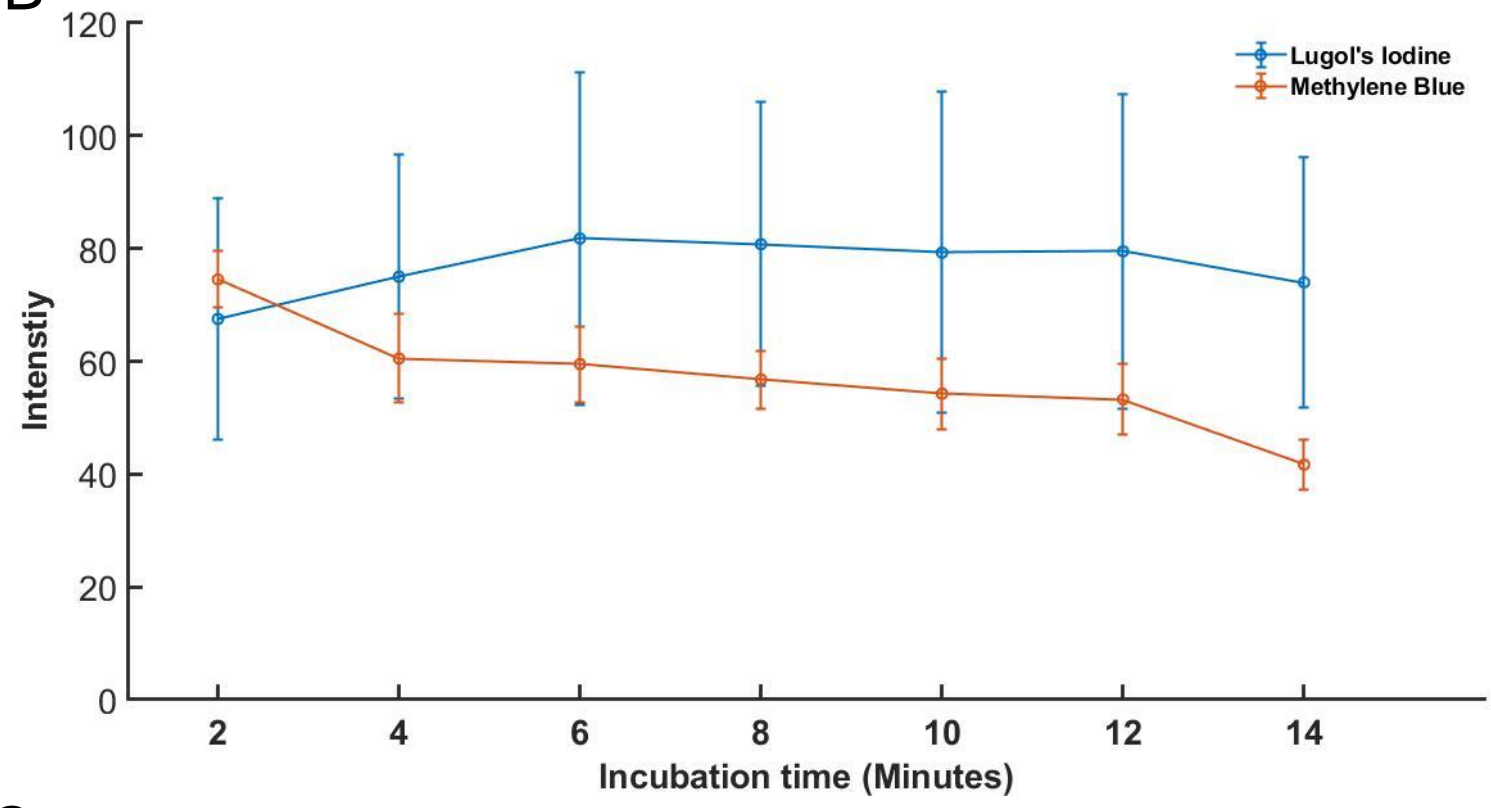

C

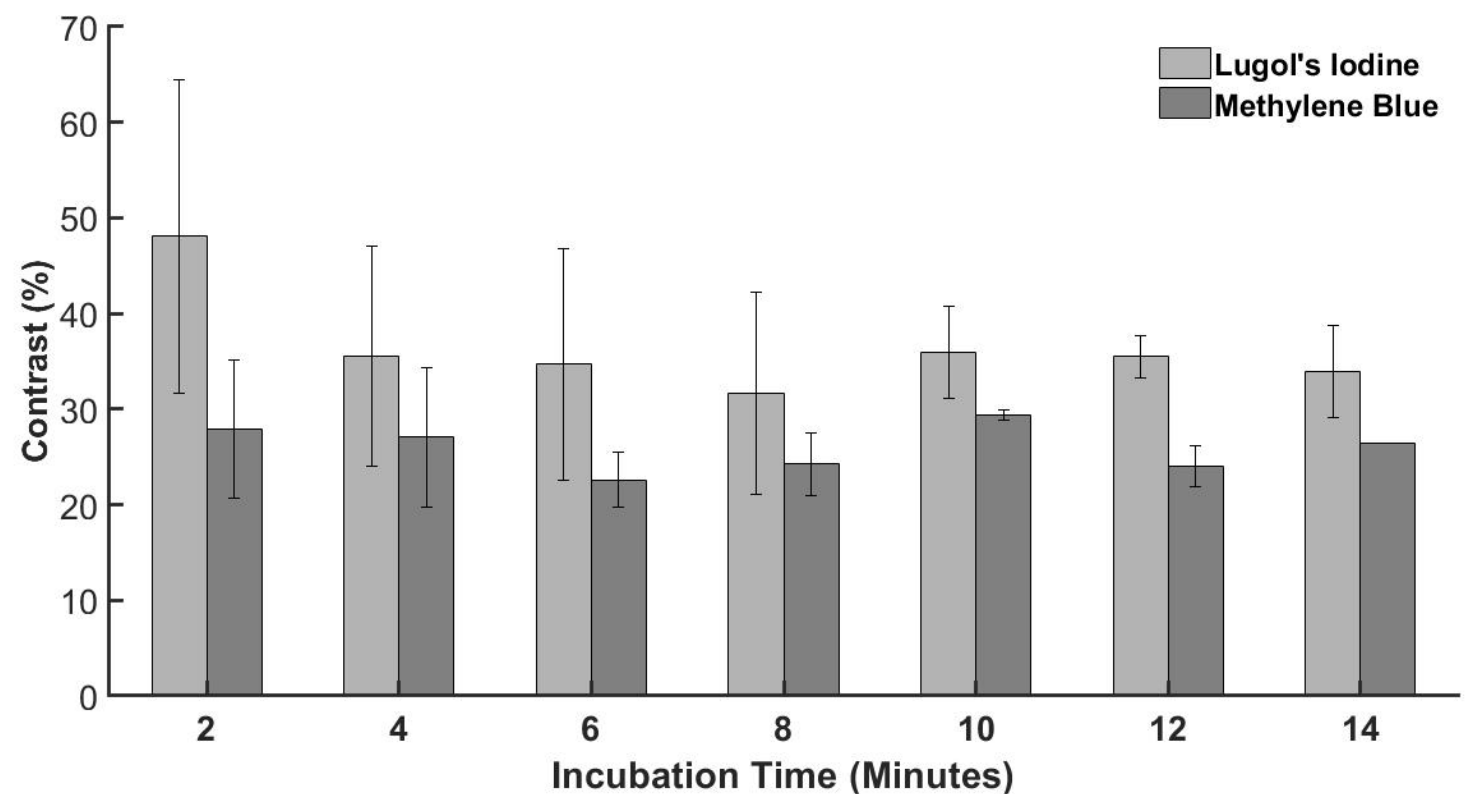

Figure 3: Representative images of Lugol's iodine and methylene blue staining (A). Both of the images were taken at 10 min of staining. The average intensity of (oo)cysts under white 
LED light source at different time intervals (B). A plot of contrast versus incubation time for methylene blue (MB) and Lugol's iodine staining (C).

\section{Method validation}

Accuracy of the smartphone microscope was evaluated by spike recovery experiments using both vegetable and water samples. In this experiment, known number of (oo)cyst were spiked to the sample and the number of (oo)cyst recovered were counted with the smartphone microscope. We compared the results from smartphone microscope with measurements using commercial bright field and fluorescence microscopes (Table 2).

Table 2: Percentage recovery of Cryptosporidium and Giardia using smartphone, commercial brightfield, and fluorescence microscopes.

\begin{tabular}{|c|c|c|c|c|c|c|}
\hline \multirow{3}{*}{ Sample } & \multicolumn{6}{|c|}{ Recovery (\%) } \\
\hline & \multicolumn{2}{|c|}{ Smartphone microscope } & \multicolumn{2}{|c|}{ Brightfield microscope } & \multicolumn{2}{|c|}{ Fluorescence microscope } \\
\hline & Cryptosporidium & Giardia & Cryptosporidium & Giardia & Cryptosporidium & Giardia \\
\hline Cabbage & $26.8 \pm 10.3$ & $10.2 \pm 4.0$ & $40.2 \pm 7.1$ & $21.6 \pm 6.7$ & $21.2 \pm 3.2$ & $18.4 \pm 2.6$ \\
\hline Carrot & $40.1 \pm 8.5$ & $14.1 \pm 7.3$ & $62.7 \pm 15.8$ & $24.7 \pm 11.4$ & $22.8 \pm 5.5$ & $20.1 \pm 6.3$ \\
\hline Cucumber & $44.4 \pm 7.3$ & $24.2 \pm 12.1$ & $51.9 \pm 18.3$ & $26.7 \pm 10.6$ & $22.8 \pm 3.5$ & $19.9 \pm 4.1$ \\
\hline Radish & $47.6 \pm 11.3$ & $23.2 \pm 13.7$ & $60.3 \pm 12.3$ & $26.7 \pm 11.9$ & $23.9 \pm 5.0$ & $20.4 \pm 2.9$ \\
\hline Tomato & $49.2 \pm 10.9$ & $17.1 \pm 13.9$ & $58.3 \pm 14.3$ & $21.7 \pm 9.0 \%$ & $32.3 \pm 6.6$ & $29.2 \pm 2.8$ \\
\hline Water & $30.2 \pm 7.9$ & $37.6 \pm 2.4$ & $35.3 \pm 7.0$ & $45.6 \pm 6.6$ & $44.4 \pm 8.8$ & $55.8 \pm 7.4$ \\
\hline
\end{tabular}

The recovery of Giardia ranged from $10.2 \pm 4.0 \%$ in cabbage to $37.6 \pm 2.4 \%$ in water and recovery of Cryptosporidium ranged from $26.8 \pm 10.3 \%$ in cabbage to $49.2 \pm 10.9 \%$ in tomato using smartphone microscope measurement (see Table 2). For most of samples, the percentage recovery was found to be marginally higher in bright field microscopy than in smartphone microscopy. The recovery of oocyst was higher than the cyst in all three microscopes with few exceptions. In image plane (in camera), the smartphone microscope has circular field of view having diameter of $\sim 200 \mu \mathrm{m}$, whereas the commercial bight filed microscope at 400x has rectangular field of view of $\sim 190 \mu \mathrm{m} \times 350 \mu \mathrm{m}$. The lower percentage recovery in smartphone 
than in brightfield microscope is mostly likely due to lower field of view which makes (oo)cyst counting difficult. The percentage recovery data reported in this work are comparable to literature studies. Cook et al. [30] reported the percentage recovery of Giardia and Cryptosporidium in spiked lettuce and raspberries of $30.4 \%$. and $44.3 \%$, respectively. In another study, Cook et al., [31] developed a method for detection of Giardia cysts and Cryptosporidium oocysts on lettuces and other salad products. They used texas red dye to stain Giardia cyst and Cryptosporidium oocyst that yielded recoveries on a variety of commercially available natural foods of $36.5 \pm 14.4 \%$ and $36.2 \pm 19.7 \%$, respectively. Similarly, in a study conducted by Amoro et al. [25] in 19 salad products, following the same method of Cook et al. [30-32], recoveries of the texas red-stained Cryptosporidium and Giardia were 24.5 $\pm 3.5 \%$ and $16.7 \pm 8.1 \%$ respectively.

The recovery efficiencies varied in certain percentages among all five different vegetables. The average recovery efficiency of (oo) cysts of Giardia by both brightfield microscopy and smartphone microscopy was higher in radish and cucumber. On the other hand, the bright field microscopy showed more Cryptosporidium in carrot and radish whereas, it was higher in radish and tomato by smartphone microscopy. The highest recovery efficiency was observed in tomato followed by radish, carrot, cucumber and cabbage for both Giardia and Cryptosporidium by fluorescence microscopy (Table 2). This difference in recovery efficiencies in various vegetables using the same methodology may be due to the variability of the noncovalent interactions between (oo) cyst surfaces and surfaces of various vegetables we tested. It is also important to note that the extraction methods that have been proven suitable for one specific food matrix could be unsuitable for the others [31-34]. For example, the glycine wash buffer had satisfactorily recovered both Giardia and Cryptosporidium in lettuce and raspberries [13, 31-33]. On the other hand, a prolonged, vigorous washing of Spinacia oleracea [35] and apples [36] in $1 \mathrm{M}$ glycine ( $\mathrm{pH}$ 5.5) elution buffer was not able to remove all of the Cryptosporidium oocysts from their matrix $[35,36]$.

For comparison, we also performed spike recovery experiments with a commercial florescent microscope. The highest recovery was observed in tomato samples for Cryptosporidium $(32.3 \pm 6.5 \%, \mathrm{n}=15)$ and for Giardia $(29.3 \pm 2.8 \%, \mathrm{n}=15)$ which was followed by radish samples with $(23.9 \pm 5.4 \%, \mathrm{n}=15)$ for Cryptosporidium and $(20.4 \pm 2.9 \%, \mathrm{n}=15)$ for Giardia (Table 2). The recovery efficiency was consistently lowest for all the samples with the fluorescence microscopy except for the tomatoes and water samples, in comparison to the smartphone and bright field microscopy. The recovery using fluorescence microscope, in which the (oo)cysts 
were tagged with fluorescent dye tagged antibody, was found to be lower than in remaining two microscopes, except in water samples. Fluorescence microscopy is dependent on binding of the fluorescence tagged antibodies to the antigen surface, which can be hindered/altered by the impurities present in the solution like the vegetable debris in our case. In some cases, fluorescent antibody could bind to the impurities and show the false positive. This could be an explanation for the higher fluorescence in the sample purified from the tomatoes. In addition of hindering binding of antibodies to oo(cysts), the larger particles can deposit (oo)cysts underneath so that they are no more accessible for antibodies. So, lower detection in case of radish, cabbage, cucumber and carrot could be due to decreased binding affinity of the antibody to the (oo)cysts or hiding of (oo) cysts underneath the larger vegetable debris [37].

Table 2 also lists the recovery efficiencies of the spiked water samples, detected by the smartphone microscope with a commercial bright filed microscope and fluorescence

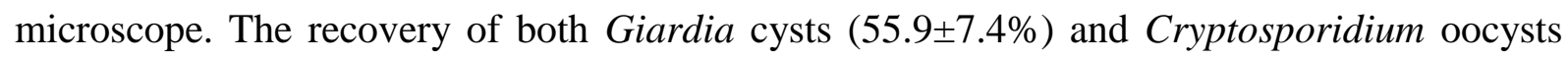
$(44.4 \pm 8.8 \%)$ were higher in fluorescence microscopes compared to both smartphone and bright field microscopes. For smartphone microscope, 37.6 $\pm 2.4 \%$ cysts and $30.2 \% \pm 7.9 \%$ oocysts were observed whereas it was $45.6 \pm 6.6 \%$ cysts and $35.3 \pm 7.0 \%$ oocysts in bright field microscopy.

Previous studies have reported similar percentage recovery data. Le Chevallier et al. used the immunofluorescence microscopic method and reported an average recovery efficiency of 68.6\% for Giardia cysts and $25.3 \%$ for Cryptosporidium oocysts in seeded tap water [38] . In another study Le Chvallier et al. showed a recovery of $96 \%$ and $77 \%$ for cysts and oocysts respectively, with the Percoll sucrose density gradient at a specific gravity $\geq 1.10$ [39]. Koompapong et al. using a similar methodology reported a recovery of oocysts (75\%) in water samples [40]. In contrast, Machado et al. found a significantly small recovery of $5.3 \%$, who analyzed the sediment of water samples using Kinyoun and Koster histochemical staining techniques [41]. They didn't use any chemical precipitant for the flocculation of oocysts before purification steps. Karanis et al (2001) compared different flocculants and concluded that using ferric sulfate yield a higher recovery $(61.5 \%)$ of $C$. parvum oocysts from tap water with a very low impact on the viability of oocysts [27]. Also, no detergent solutions were included in the study that helped to set the oocysts free from the sediments [41]. In a study made by Hsu et al. standard Envirochek capsule filtration followed by immunomagnetic separation, the standard purification procedure in Environmental Protection Agency Method 1623, was used. In their 
study, the recovery efficiencies were higher for Giardia (48.0\%) than for Cryptosporidium $(32 \%)$ [42]. These data are very similar to our percentage recovery data in water samples.

We also estimated method detection limit (LOD) of smartphone microscope method. The LOD varied with type of sample. LOD of Giardia ranged from 24 cyst/100 g for cucumber to 73 cyst $/ 100 \mathrm{~g}$ for cabbage (tomato $=38$, carrot $=40$ and cucumber $=23 \mathrm{cyst} / 100 \mathrm{~g}$ ). Similarly, the LOD for Cryptosporidium ranged from 11 oocyst/100 g for radish to 25 oocyst/100 g for cabbage (tomato $=12$, carrot $=12$ and cucumber $=23$ oocyst $/ 100 \mathrm{~g}$ ). In general, the LOD of Cryptosporidium was lower than that of Giardia.

\section{Prevalence of (oo)cysts in vegetable and water samples}

After developing the smartphone microscopic system for (oo)cyst detection, we screened (oo)cysts contamination in five different types of vegetable samples $(n=196)$ purchased from local market in Kathmandu, Nepal and river water samples $(n=18)$. The samples were processed as described in method section.

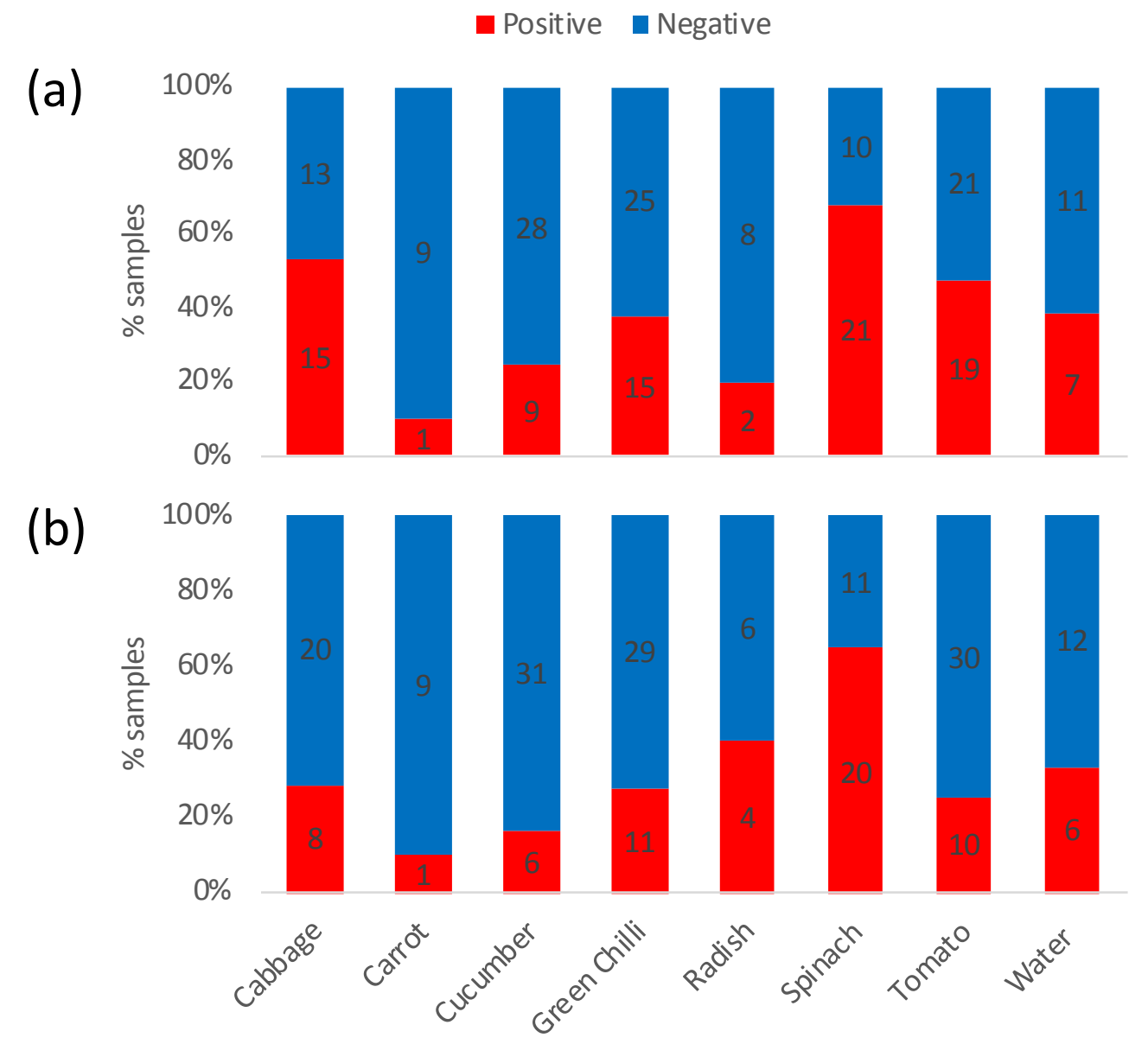

Figure 4: Percentage of presence or absence of cyst in each sample types using smartphone microscope: a) Cryptosporidium and b) Giardia. The numbers in each bar graph represent the number of samples. 
The result is shown in figure 4 . Out of the total samples, $31.1 \%$ vegetable samples were positive for cyst and $35.2 \%$ samples were positive for oocyst contamination when detected using smartphone microscope. The prevalence of oocysts was highest in spinach samples and lowest in carrot samples (see Figure 4). The same samples were also tested using brightfield microscope and fluorescence microscope and compared with smartphone microscope. The comparison is provided in Table 3. Cysts were detected in $40.3 \%$ samples oocysts were detected in $41.8 \%$ vegetable samples using brightfield microscope. Further, out of 196 samples, randomly selected $58(30 \%)$ samples were screened using fluorescence microscope. The fluorescence measurement showed that 27(46.5\%) samples were contaminated with cyst and $26(44.8 \%)$ samples were contaminated with oocyst. The shape and surface of vegetables might play a role leading to the contamination. The (oo)cysts can easily attach to the uneven or curly surfaces of spinach and cabbage either in the farm or when washed with polluted water. On the other hand, vegetables with smooth surfaces such as radishes and carrots had a low number of (oo)cysts in the present study as its smooth surface reduces the attachment of the protozoans [44].

According to a survey conducted by Maikai et al. $40 \%$ of Spinach, $32 \%$ of tomato, $24 \%$ of carrot and $16 \%$ of cabbage were contaminated with Cryptosporidium oocysts using microscopy [45]. A study by Kaudah et al. reported that $50 \%$ tomatoes, $43.1 \%$ cabbage and $26.4 \%$ of carrot were tested positive for different protozoans among which $11 \%$ were Cryptosporidium. These samples were stained with Lugol's iodine and observed under light and fluorescence microscope [46]. In contrast Utaaker et al. reported that only 14\% (8/56) tomatoes and $9 \%$ (4/47) cabbages were contaminated with either Giardia cysts or Cryptosporidium oocysts [22]. In case of root vegetables such as carrot, the current study has a very low record (10\%) for both Giardia and Cryptosporidium. Similar results were also reported in other studies like 14\% positive cases in India [22] and 6.4\% in Southern Ethiopia [47] for Giardia. A slightly higher positive cases was observed in Egypt (43.3\%) [44] and Korea (33.3\%).

We tested eighteen surface water samples collected from 3 different sites of the Bishnumati river, Kathmandu, Nepal in two different field campaigns. The samples were flocculated and purified with sucrose density gradient and examined by smartphone, commercial bright field, and fluorescence microscopies. A total of $33.3 \%$ (6 out of 18 samples) were tested positive for Giardia and seven samples (38.9\%) were tested positive for Cryptosporidium by smartphone microscope (figure 4). When compared to other microscopes, in general, higher number samples were tested positive for (oo)cysts by the smartphone microscopy. Brightfield 
microscope confirmed 22.2\% (4 out of 18) positive results for Giardia and 33.3\% (6 out of 18) positive results for Cryptosporidium. Similarly, four (22.2\%) were tested positive by for (oo)cysts using fluorescence microscopy (see Table 3).

Table 3: Prevalence of (oo)cyst contamination in vegetable and water samples measured by three different microscopic methods.

Number of samples

\begin{tabular}{|c|c|c|c|c|c|c|c|}
\hline \multirow{2}{*}{\multicolumn{2}{|c|}{ Sample type }} & \multirow{2}{*}{\multicolumn{2}{|c|}{$\begin{array}{l}\text { Smartphone } \\
\text { microscope }\end{array}$}} & \multirow{2}{*}{\multicolumn{2}{|c|}{$\begin{array}{l}\text { Brightfield } \\
\text { microscope }\end{array}$}} & \multirow{2}{*}{\multicolumn{2}{|c|}{$\begin{array}{l}\text { Fluorescence } \\
\text { microscope }\end{array}$}} \\
\hline & & & & & & & \\
\hline & & Crypto & Giardia & Crypto & Giardia & Crypto & Giardia \\
\hline \multirow[t]{2}{*}{ Cabbage } & positive & 15 & 8 & 9 & 11 & 6 & 4 \\
\hline & negative & 13 & 20 & 19 & 17 & 9 & 5 \\
\hline \multirow[t]{2}{*}{ Carrot } & positive & 1 & 1 & 1 & 1 & 1 & 1 \\
\hline & negative & 9 & 9 & 9 & 9 & 4 & 0 \\
\hline \multirow[t]{2}{*}{ Cucumber } & positive & 9 & 6 & 6 & 9 & 3 & 5 \\
\hline & negative & 28 & 31 & 31 & 28 & 8 & 4 \\
\hline Green & positive & 15 & 11 & 15 & 14 & 6 & 2 \\
\hline \multicolumn{8}{|l|}{ Chilli } \\
\hline \multirow[t]{2}{*}{ Radish } & positive & 2 & 4 & 2 & 3 & 0 & 3 \\
\hline & negative & 8 & 6 & 8 & 7 & 0 & 0 \\
\hline \multirow[t]{2}{*}{ Spinach } & positive & 21 & 20 & 20 & 21 & 6 & 6 \\
\hline & negative & 10 & 11 & 11 & 10 & 8 & 10 \\
\hline \multirow[t]{2}{*}{ Tomato } & positive & 19 & 10 & 15 & 20 & 3 & 4 \\
\hline & negative & 21 & 30 & 25 & 20 & 8 & 5 \\
\hline
\end{tabular}




\begin{tabular}{ccccccccc} 
Water & positive & 7 & 6 & 6 & 4 & 4 & 4 \\
& negative & 11 & 12 & 12 & 14 & 14 & 14 \\
\hline
\end{tabular}

We estimated the (oo)cysts per unit of sample. The highest concentration of the (oo)cysts for both Giardia and Cryptosporidium were detected in cabbages $(\mathrm{n}=27)$ with the concentration of 442 cysts and 225 oocysts $/ \mathrm{kg}$. The lowest concentration of 35 cysts $/ \mathrm{kg}$ and 16 oocysts/kg was found in radish. Tomatoes $(n=40)$, carrots $(n=10)$, and cucumbers $(n=37)$ were found to be contaminated with 129 cysts/kg, 166 cysts/kg, and, 77 cysts $/ \mathrm{kg}$, respectively. Similarly, 76 oocysts/kg, 47 oocysts/kg, 185 oocysts $/ \mathrm{kg}$, and were found in tomato $(\mathrm{n}=40)$, carrots $(\mathrm{n}=10)$, and cucumber $(\mathrm{n}=37)$, samples. The infectious dose for cryptosporidiosis and giardiasis is as low as 10-30 viable (oo)cyst [48]. Assuming around $200 \mathrm{~g}$ of poorly washed raw vegetable is consumed per day, there is still high chance that most of the samples tested were infectious if ingested.

\section{Conclusions}

We designed a smartphone microscope and optimized its various optical parameters. The field of view increases with the diameter of sapphire ball lens but the magnification follows the opposite trend; in agreement with theory. We found that microscope having ball lens of $1 \mathrm{~mm}$ diameter along with Lugol's iodine staining and commercially available white LED illumination can simultaneously determine (oo)cyst of Cryptosporidium and Giardia in vegetable samples. The spiking recovery experiment on the different vegetable and water samples showed that the $\%$ recovery is comparable to the commercial bright field microscope and better than fluorescence microscopic measurement. We found that \% recovery varied with the nature of sample and recovery for Cryptosporidium oocyst is better than Giardia cyst. This observation is consistent with the literature studies. Out of the 196 vegetable samples $31.1 \%$ vegetable samples were positive for cyst and $35.2 \%$ samples were positive for oocyst contamination when examined by smartphone microscope. This study shows that the smartphone based microscopic assay can a low-cost alternative for pre-screening of (oo)cyst of Cryptosporodium and Giardia in resource limited settings. Our future work involves the development of an automated smartphone program that could take image, process the image to identify and count the (oo)cysts, and provide report to the user. This automated system may minimize error and shorten the analysis time. 


\section{Acknowledgments}

This work was supported by NAS and USAID through Partnerships for Enhanced Engagement in Research (PEER) (AID-OAA-A-11-00012). The opinions, findings, conclusions, or recommendations expressed in this article are those of the authors alone, and do not necessarily reflect the views of USAID or NAS. 


\section{References}

1. Buzby, J.C., Effects of food-safety perceptions on food demand and global trade. Changing structure of global food consumption and trade, 2001: p. 55-66.

2. $\quad$ Organization, W.H., Food Safety. 2019.

3. Law, J.W.-F., et al., Rapid methods for the detection of foodborne bacterial pathogens: principles, applications, advantages and limitations. Frontiers in microbiology, 2015. 5: p. 770.

4. Organization, W.H., Multicriteria-based ranking for risk management of food-borne parasites: report of a Joint FAO. 2014.

5. Dixon, B., et al., Protozoan parasites: Cryptosporidium, Giardia, Cyclospora, and Toxoplasma, in Rapid detection, characterization, and enumeration of foodborne pathogens. 2011, American Society of Microbiology. p. 349-370.

6. Smith, J.L., Cryptosporidium and Giardia as agents of foodborne disease. Journal of Food Protection, 1993. 56(5): p. 451-461.

7. Quah, S.R., International encyclopedia of public health. 2016: Academic Press.

8. Molloy, S., et al., Sporadic Cryptosporidium infection in Nigerian children: risk factors with species identification. Epidemiology \& Infection, 2011. 139(6): p. 946-954.

9. Mejia, R., et al., A novel, multi-parallel, real-time polymerase chain reaction approach for eight gastrointestinal parasites provides improved diagnostic capabilities to resource-limited at-risk populations. The American journal of tropical medicine and hygiene, 2013. 88(6): p. 10411047.

10. Ali, M., A. Al-Herrawy, and S. El-Hawaary, Detection of enteric viruses, Giardia and Cryptosporidium in two different types of drinking water treatment facilities. Water Research, 2004. 38(18): p. 3931-3939.

11. Hsu, B.-M., et al., Using the flow cytometry to quantify the Giardia cysts and Cryptosporidium oocysts in water samples. Environmental monitoring and assessment, 2005. 104(1-3): p. 155162.

12. Hill, V.R., et al., Comparison of hollow-fiber ultrafiltration to the USEPA VIRADEL technique and USEPA method 1623. Journal of Environmental Quality, 2009. 38(2): p. 822-825.

13. Slusarewicz, P., et al., Automated parasite faecal egg counting using fluorescence labelling, smartphone image capture and computational image analysis. International journal for parasitology, 2016. 46(8): p. 485-493.

14. Coskun, A.F., et al., A personalized food allergen testing platform on a cellphone. Lab on a Chip, 2013. 13(4): p. 636-640.

15. Breslauer, D.N., et al., Mobile phone based clinical microscopy for global health applications. PloS one, 2009. 4(7): p. e6320.

16. Wei, Q., et al., Fluorescent imaging of single nanoparticles and viruses on a smart phone. ACS nano, 2013. 7(10): p. 9147-9155.

17. D'Ambrosio, M.V., et al., Point-of-care quantification of blood-borne filarial parasites with a mobile phone microscope. Science translational medicine, 2015. 7(286): p. 286re4-286re4.

18. Agbana, T.E., et al., Imaging \& identification of malaria parasites using cellphone microscope with a ball lens. PloS one, 2018. 13(10): p. e0205020.

19. Smith, Z.J., et al., Cell-phone-based platform for biomedical device development and education applications. PloS one, 2011. 6(3): p. e17150.

20. Bogoch, I.I., et al., Mobile phone microscopy for the diagnosis of soil-transmitted helminth infections: a proof-of-concept study. The American journal of tropical medicine and hygiene, 2013. 88(4): p. 626-629.

21. Peli, E., Contrast in complex images. JOSA A, 1990. 7(10): p. 2032-2040.

22. Utaaker, K.S., et al., Checking the detail in retail: occurrence of Cryptosporidium and Giardia on vegetables sold across different counters in Chandigarh, India. International journal of food microbiology, 2017. 263: p. 1-8. 
23. Kozan, E., et al., Prevalence of helminth eggs on raw vegetables used for salads. Food Control, 2005. 16(3): p. 239-242.

24. Rahman, J., et al., Detection of Cryptosporidium oocyts in commonly consumed fresh salad vegetables. American Journal of Microbiological Research, 2014. 2(6): p. 224-226.

25. Amorós, I., J.L. Alonso, and G. Cuesta, Cryptosporidium oocysts and Giardia cysts on salad products irrigated with contaminated water. Journal of food protection, 2010. 73(6): p. 11381140.

26. Medeiros, R.C. and L.A. Daniel, Comparison of selected methods for recovery of Giardia spp. cysts and Cryptosporidium spp. oocysts in wastewater. Journal of water and health, 2015. 13(3): p. 811-818.

27. Karanis, P. and A. Kimura, Evaluation of three flocculation methods for the purification of Cryptosporidium parvum oocysts from water samples. Letters in applied microbiology, 2002. 34(6): p. 444-449.

28. Stender, A.S., et al., Single cell optical imaging and spectroscopy. Chemical reviews, 2013. 113(4): p. 2469-2527.

29. Cybulski, J.S., J. Clements, and M. Prakash, Foldscope: origami-based paper microscope. PloS one, 2014. 9(6): p. e98781.

30. Cook, N., et al., Towards standard methods for the detection of Cryptosporidium parvum on lettuce and raspberries. Part 2: validation. International journal of food microbiology, 2006. 109(3): p. 222-228.

31. Cook, N., et al., Development of a method for detection of Giardia duodenalis cysts on lettuce and for simultaneous analysis of salad products for the presence of Giardia cysts and Cryptosporidium oocysts. Appl. Environ. Microbiol., 2007. 73(22): p. 7388-7391.

32. Cook, N., et al., Towards standard methods for the detection of Cryptosporidium parvum on lettuce and raspberries. Part 1: development and optimization of methods. International journal of food microbiology, 2006. 109(3): p. 215-221.

33. Hohweyer, J., et al., Simultaneous detection of the protozoan parasites Toxoplasma, Cryptosporidium and Giardia in food matrices and their persistence on basil leaves. Food microbiology, 2016. 57: p. 36-44.

34. Robertson, L. and B. Gjerde, Isolation and enumeration of Giardia cysts, Cryptosporidium oocysts, and Ascaris eggs from fruits and vegetables. Journal of food protection, 2000. 63(6): p. 775-778.

35. Macarisin, D., G. Bauchan, and R. Fayer, Spinacia oleracea L. leaf stomata harboring Cryptosporidium parvum oocysts: a potential threat to food safety. Appl. Environ. Microbiol., 2010. 76(2): p. 555-559.

36. Macarisin, D., et al., Infectivity of Cryptosporidium parvum oocysts after storage of experimentally contaminated apples. Journal of Food Protection, 2010. 73(10): p. 1824-1829.

37. Ganz, K.R., et al., Enhancing the detection of Giardia duodenalis cysts in foods by inertial microfluidic separation. Appl. Environ. Microbiol., 2015. 81(12): p. 3925-3933.

38. LeChevallier, M.W., W.D. Norton, and R.G. Lee. Evaluation of a method to detect Giardia and Cryptosporidium in water. in Monitoring Water in the 1990's: Meeting New Challenges. 1991. ASTM International.

39. LeChevallier, M.W., et al., Evaluation of the immunofluorescence procedure for detection of Giardia cysts and Cryptosporidium oocysts in water. Appl. Environ. Microbiol., 1995. 61(2): p. 690-697.

40. Koompapong, K., C. Sutthikornchai, and Y. Sukthana, Cryptosporidium oocyst detection in water samples: floatation technique enhanced with immunofluorescence is as effective as immunomagnetic separation method. The Korean journal of parasitology, 2009. 47(4): p. 353.

41. Machado, E., et al., Effectiveness of Cryptosporidium spp. oocysts detection and enumeration methods in water and milk samples. Arquivo Brasileiro de Medicina Veterinária e Zootecnia, 2006. 58(3): p. 432-439. 
42. Hsu, B.-M. and C. Huang, Recovery of Giardia and Cryptosporidium from water by various concentration, elution, and purification techniques. Journal of environmental quality, 2000. 29(5): p. 1587-1593.

43. Shepherd, K. and A. Wyn-Jones, An evaluation of methods for the simultaneous detection of Cryptosporidium oocysts and Giardia cysts from water. Appl. Environ. Microbiol., 1996. 62(4): p. 1317-1322.

44. El Said Said, D., Detection of parasites in commonly consumed raw vegetables. Alexandria Journal of Medicine, 2012. 48(4): p. 345-352.

45. Maikai, B., E. Baba-Onoja, and I. Elisha, Contamination of raw vegetables with Cryptosporidium oocysts in markets within Zaria metropolis, Kaduna State, Nigeria. Food Control, 2013. 31(1): p. 45-48.

46. Kudah, C., S. Sovoe, and F. Baiden, Parasitic contamination of commonly consumed vegetables in two markets in Ghana. Ghana medical journal, 2018. 52(2): p. 88-93.

47. Alemu, G., et al., Parasitic contamination of vegetables marketed in Arba Minch town, southern Ethiopia. BMC infectious diseases, 2019. 19(1): p. 410.

48. Yoder, J.S., et al., Cryptosporidiosis surveillance-United States, 2003-2005. MMWR Surveill Summ, 2007. 56(7): p. 1-10. 